\title{
SUMMARY OF THE SERIES OF ARTICLES ON THE VITAMINS.
}

\author{
By SiR JOHN BOYD ORR, M.D., D.Sc., LL.D., F.R.S.
}

(Director, The Rowett Research Institute, Aberdeenshire.)

\section{The Role of Vitamin Therapy in Medicine.}

Therapeutic dietetics has become such an important branch of medicine that the clinician must keep himself informed of recent advances on the subject. But it is impossible for him to read the enormous literature amounting, as it does, to an annual output of about 5,000 papers, of which about half deal with vitamins. This series of reviews should prove of value in providing a statement on the present knowledge of vitamins of importance in medicine.

In considering the role vitamin therapy may play in medicine, it is necessary to realise that vitamins should not be regarded as drugs. They are essential constituents of the diet but no more essential than energy-yielding constituents or inorganic salts. If the supply in the diet is sufficient to maintain the normal physiological functions with which they are concerned, no benefit will be derived from additional amounts. Rational vitamin therapy, therefore, depends upon the recognition of the signs of deficiency or a knowledge of a pre-existing deficiency.

In medical practice, it is not always easy to diagnose vitamin deficiency. Our knowledge of the signs of deficiency has been obtained largely from research on experimental animals, standardised in their hereditary make-up, kept under uniformly good environmental conditions, and reared, as far as possible, free from other disease. Further, the composition of the diets used is usually adjusted to be lacking in only one vitamin at a time. In human populations, such conditions are never reproduced. A diet that is deficient in one substance is usually deficient in many. If there are obvious signs of one deficiency, symptomatic treatment with the vitamin concerned will, no doubt, remove them, but those of the deficiency of other dietary constituents may be unmasked. While, therefore, in cases of gross deficiency, the effects of the constituent most deficient are usually easily recognised, in most cases met with in practice there is likely to be a mixture of signs and symptoms of several deficiencies made more confused by the after effects of previous or the presence of concurrent infectious disease. The picture may be further confused by psychological factors. Thus, for example, the nervous, cardiac or gastric symptoms of deficiency of $\mathrm{B}_{1}$ may be indistinguishable clinically from symptoms which may have a psychological origin. Hence, though clinical research is rapidly increasing our knowledge of the signs and symptoms of minor degrees of deficiency, the number of other factors which may be partly responsible for the ill-health of the patient makes the clinical diagnosis of vitamin deficiency extremely difficult.

More specific indications of unknown deficiencies can, of course, now be obtained by chemical tests of body fluids and tests of efficiency of physiological functions. The technique of these tests has been greatly improved in recent years and, though there is not yet complete unanimity on the full significance of the data obtained, there can be no doubt that they have considerable diagnostic value and are likely to be used to an increasing extent in future. The correlation of the data of these tests with the results of clinical examination with subsequent observations on the effect of treatment is probably the most promising line of advance in improving methods of diagnosis.

Though the clinician be fully equipped with these methods of diagnosis, his judgment will be largely influenced by his knowledge or suspicion of previous deficiency. It is, therefore, necessary to consider to what extent and under what circumstances sub-acute vitamin deficiency occurs. This question cannot be answered without an estimate of 
requirements. These can be stated only within rather wide limits. It is possible to define, for some of the vitamins, lower limits below which clinical signs of deficiency are likely to appear, but no high degree of accuracy attaches to these estimates. Individual variation, differences in requirements due to rate of growth, pregnancy and lactation, infectious disease and other complications, confuse the picture. Nor is the lower limit the more important. It is much more important to know the requirement for perfect health. The estimates of requirements for health which have been made are based on a great mass of data derived from experiments on animals, feeding tests with children and clinical observations. Though they may be modified by the results of further work, the most recent estimates can be taken as a fairly reliable guide to practice. It is more difficult to estimate the intake. Natural foods vary considerably in their vitamin content and there is a variable loss in storage, preparation and cooking. Taking account of these factors, estimates have been made from the results of dietary surveys in this and other countries. A comparison of the estimated average intake with requirements indicates that the diet of large numbers of families is deficient in some of the vitamins. While deficiency is much more marked among the poor, it is not uncommon among well-to-do families. It is by no means safe to assume that because a child comes from a well-to-do family its diet can be considered fully adequate. In the course of our dietary and clinical survey of children carried out from this Institute, we have had many instances of deficiencies in the diet with corresponding clinical signs in children from homes where faulty diet could not be attributed to poverty.

The most direct method of determining whether there is a deficiency in the diet is to increase the vitamin intake and note whether or not there is an improvement in health. The results of numerous well-controlled feeding experiments with school children show that increased consumption of vitamin-rich foods is followed, in a large majority of cases, by improved health and vigour. There are also many reports of excellent results from vitamin therapy in conditions not previously regarded as of dietary origin and in cases of indefinite ill-health of obscure etiology. But it must be remembered that, in clinical medicine, many of these therapeutic successes are uncontrolled experiments and there is danger of mistaking post hoc for propter hoc. Further, in the feeding experiments with protective foods, the supplements supply more than vitamins. They are rich in protein and essential minerals which are usually deficient in poor diets. It is impossible, therefore, to attribute the whole of the effect to vitamins, although there is no doubt that they play an important part.

The position then seems to be that the data of dietary surveys, the results of feeding tests and the frequent beneficial results of vitamin therapy, all indicate that, in spite of the great increase in the consumption of protective foods in recent years, minor degrees of vitamin deficiency are still common. If this be the case, as many of us believe, then in a large proportion of children presented for treatment vitamin deficiency may be a contributory cause of the illness. In many cases of indefinite ill-health of obscure etiology, a deficiency may be the primary cause, though, owing to the difficulties of clinical diagnosis, it is often impossible to be certain that vitamin deficiency is the cause of the condition. Even in cases in which the main cause of the illness is obvious and not connected with faulty diet, vitamin therapy may play a part in the treatment. In certain febrile and in rheumatic conditions, the need for vitamin $\mathrm{C}$ seems to be increased and it is probable that, in many other pathological conditions, an intake of some of the vitamins greater than normal requirements may be called for, though, indeed, on this subject, which is only beginning to be investigated, we have, as yet, comparatively little information. In these circumstances, it would appear that a careful regulation of the diet to ensure an adequate intake of vitamins should form an important part of treatment in practically all cases of sickness. 
Even when, from the signs and symptoms, a deficiency of one of the vitamins can be diagnosed, it is not sufficient merely to administer the appropriate vitamin concentrate. It cannot be too strongly emphasised that, if there is a deficiency of one vitamin sufficient to cause the appearance of pathological signs and symptoms, it is practically certain that the diet will be faulty in other respects. Therefore, if a deficiency of one essential be made good, the lack of another immediately becomes the limiting factor for health. It is recorded that the cure of beriberi by pure vitamin $B_{1}$ without any change of diet has been followed by the immediate development of pellagra. It is a mistake, therefore, to trust entirely to vitamin concentrates. The fundamental part of the treatment is to make the whole diet adequate and then, if necessary, to fortify by the appropriate concentrates.

In giving advice on dietetics, in many cases it may be necessary for a time to set down a prescribed diet. But it is unnecessary for normal healthy children to eat according to a schedule. Indeed, the requirements of different children vary and even the requirements of the same child vary from time to time. The League of Nations Report on Physiological Bases of Nutrition (1935, 1936) and the booklet "Food for Health in Peace and War," prepared by the Canadian Medical Association (I940) give examples of diets which are adequate. But these are only examples. As a general rule, adequacy will be attained for children when about two-thirds of the total calories supplied are provided by a mixed group of protective foods, including milk. Under the present food restrictions due to the war, some protective foods, such as eggs and fruit, are not obtainable in amounts sufficient to fulfil the prescriptions of these diets, planned in peace-time. Fortunately, diets, adequate in vitamins as well as minerals and protein, can be provided with milk, green vegetables and wholemeal bread or oatmeal as the basis.

In this general discussion of vitamins in therapeutic dietetics, I have tried to make clear two ideas. First, dietary deficiency, including vitamin deficiency, is a common primary or contributory cause of ill-health, and therefore the adjustment of the diet to make it adequate, with concentrates in special cases, where curative treatment is required, should form an important part of the treatment of many, and probably most, cases of illness in children. Second, our knowledge of the therapeutic aspect is so imperfect that there is great need for careful clinical observation of the effect of vitamin therapy in many different conditions in which it is being tried. In the present imperfect state of our knowledge, this is one of the most important fields of clinical research. It can be carried out in general practice as well as in medical schools. If the general practitioner would make careful observations and record and report the effect of treatment, he would make a contribution to the aspect of nutrition in which information is most urgently needed. If this series of papers stimulates the making and recording of such observations. it will serve a most useful purpose.

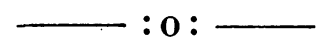

